\title{
Lecturas de Piaget en América Latina: Emilia Ferreiro, la lectoescritura y el fracaso escolar
}

\section{Leituras de Piaget na América Latina: Emilia Ferreiro, alfabetização e fracasso escolar}

\section{Piaget readings in Latin America: Emilia Ferreiro, literacy and school failure}

\author{
Patricia Scherman* \\ Universidad Nacional de Córdoba - UNC, Córdoba, Argentina \\ Laura Vissani** \\ Universidad Nacional de Córdoba - UNC, Córdoba, Argentina \\ Nilda Fantini*** \\ Universidad Nacional de Córdoba - UNC, Córdoba, Argentina
}

\begin{abstract}
RESUMEN
Se presentan las transformaciones en la lectura y utilización de la teoría de Piaget que desarrollaron en la Argentina en la década de 1970, y cómo estas recogieron importantes problemáticas sociales de su tiempo. En primer lugar se presenta la labor de Emilia Ferreiro, sus antecedentes formativos y la investigación que dio lugar a la publicación del libro Los sistemas de escritura en el desarrollo del niño (Ferreiro \& Teberosky, 1979). Luego se destacan las innovaciones que ese texto produjo en la época: la introducción de una lectura novedosa de la teoría de Piaget; la utilización de conceptos de psicología genética en un campo nuevo; la realización de una investigación empírica desde el método piagetiano; la localización del problema del acceso a la lectoescritura y su relación con el fracaso escolar involucrando aspectos hasta ese momento invisibilizados, como la pobreza y la desigualdad social en América Latina. Finalmente se analiza la recepción del texto en la Argentina, donde aparecen las marcas de la dictadura militar, como el exilio, la censura y la clandestinidad; el encuentro con el texto de Ferreiro muestra una cara de la persecución ejercida sobre los intelectuales y las formas clandestinas de circulación de saberes que una generación profesional enfrentó.
\end{abstract}

Palabras claves: Piaget, lectoescritura, Ferreiro, dictadura, Argentina.

\section{RESUMO}

O texto apresenta as transformações na leitura e utilização da teoria de Piaget na Argentina na década de 1970, e como foram estes trabalhos levantaram importantes questões sociais da época. Primeiro, introduz-se o trabalho de Ferreiro, sua formação educacional e as pesquisas que levaram à publicação do livro Psicogênese da língua escrita (Ferreiro \& Teberosky, 
1979). Em seguida, apresentam-se as inovações que esse texto produziu na época: uma nova leitura da teoria de Piaget na mídia acadêmica da Argentina; a utilização da psicologia genética em um novo campo; a realização de uma pesquisa empírica a partir do método piagetiano; a localização do problema do acesso à alfabetização e sua relação com o fracasso escolar, envolvendo aspectos até então invisíveis, como a pobreza e a desigualdade social na América Latina. Finalmente, expomos os avatares da recepção do texto na Argentina, aí aparecendo as marcas da ditadura militar, como o exílio, a censura e a clandestinidade; o encontro com o texto de Ferreiro mostra o rosto da perseguição exercida sobre os intelectuais e as formas clandestinas de circulação de conhecimento que uma geração profissional teve que enfrentar.

Palavras chaves: Piaget, alfabetização, Ferreiro, ditadura, Argentina.

\begin{abstract}
Transformations in readings and use of the theory of Piaget in Argentina in the 1970s are presented, and how they collected social important problems of their time. First, we present the work of Emilia Ferreiro, his educational background and the research that led to the publication of the book Literacy before schooling (Ferreiro \& Teberosky, 1979). Then we highlight the innovations that that text introduced: a novel reading of Piaget's theory in the academic media of Argentina; the qualification of genetic psychology concepts in a new field; an empirical research process from the Piagetian method; To place the problem of access to literacy and its relation to school failure, involving aspects that until then have been invisible, such as poverty and social inequality in Latin America. Finally we expose the vicissitudes of the reception in Argentina, were they appeared the marks of the military dictatorship, such as exile, censorship and clandestinity; the encounter with Ferreiro's text shows one side of the persecution exerted on the intellectuals and clandestine forms of circulation of knowledge that a professional generation had to face.
\end{abstract}

Keywords: Piaget, literacy, Ferreiro, dictatorship, Argentina.

\title{
I ntroducción
}

Esta presentación busca mostrar las transformaciones en la lectura y utilización de la teoría de Piaget en Argentina que desarrollaron Emilia Ferreiro y sus colaboradoras en la década de 1970, y cómo esas elaboraciones recogieron importantes problemáticas sociales de su tiempo, tales como el fracaso escolar y su desigual distribución en los niños de América Latina, a las que aportó nuevas coordenadas de comprensión. Nos interesa mostrar también la creatividad de las investigadoras en la utilización de postulados centrales de la teoría de Piaget articuladas en un campo novedoso -el de la lectoescritura-, y en el diseño de originales investigaciones locales.

Recuperar la originalidad de una lectura que incluyó los aspectos sociales de la problemática del fracaso escolar en América Latina, implica señalar cómo las referencias a Piaget se separan del cuadro de una psicología evolutiva abstracta y remitida a un sujeto individual. El sujeto en Emilia Ferreiro ya no será este niño de la 
psicología evolutiva, sino que se tratará de un sujeto epistémico, fuertemente condicionado por sus coordenadas sociales y económicas de vida. Es decir un sujeto cognoscente que también se convierte en social. Por otra parte, en la producción y recepción del texto se hacen presentes los efectos de la dictadura militar argentina en la interrupción de esas fértiles lecturas: en el mismo movimiento por el que se suspende una investigación psicológica, esta se expande a otros países, como Brasil y Méjico, y retorna a la Argentina de manera clandestina.

En este sentido nos preguntamos ¿Cuáles fueron los aportes novedosos introducidos por Ferreiro en el campo del aprendizaje y de lo escolar? ¿Cuáles fueron las implicancias de las investigaciones de Ferreiro para ser interrumpidas por la dictadura? ¿Cómo se relaciona su trabajo con la sombra de censura que recaía sobre la obra de Piaget en la Argentina de la dictadura?

Estas preguntas, al ser articuladas en el marco de la historia social, entendida como aquella forma de considerar la historia que toma en cuenta los fenómenos sociales como parte fundamental de la elaboración del relato histórico, permite reconocer cambios de mentalidades, de formas de entender el mundo que se despliegan en la vida cotidiana de la gente corriente (Grez Toso, 2005). En lo metodológico permite incorporar la oralidad, la participación de los propios actores, su subjetividad como una parte importante de la historia ya que trata de cómo los propios actores ven los hechos históricos, se conciben a sí mismos y conciben a los otros (Grez Toso, 2005; Casanova, 2015). Su importancia como campo específico se liga al impacto de los aportes historiográficos en la sociedad actual. “En la actualidad, la literatura crítica sobre historia de la psicología, de la psiquiatría y el psicoanálisis incluye las tramas sociales, culturales, políticas e institucionales para lograr amplitud y solidez en las indagaciones" (Macchioli, García \& Talak, 2017, p. 12).

En esta presentación, al tomar el texto de Emilia Ferreiro Los sistemas de escritura en el desarrollo del niño (Ferreiro \& Teberosky, 1979) como un producto histórico, buscamos poner al descubierto algunos aspectos de esta trama social, política, cultural de la cual emerge su trabajo y el de sus colaboradoras; poder analizar cómo desde este enfoque los postulados de Piaget se desplazan del marco de una psicología evolutiva clásica para adquirir otros sentidos posibles en un campo disciplinar de límites difusos, son algunas de las dimensiones que se ponen en juego al tratar de poner en relación el libro y el momento histórico, sin lo cual estos aportes podrían verse como un listado propio de un manual de psicología.

El trabajo de Emilia Ferreiro se ubica en un campo disciplinar que implica cruces y solapamientos entre la psicología, la psicopedagogía, la pedagogía. A su vez, el momento histórico en que la investigación se inicia es entre dictaduras, la de Onganía y más tarde el proceso 
militar que gobernó la Argentina a partir de 1976. El posicionamiento y la acción política llevada a cabo por intelectuales y universitarios en aquellos momentos enmarcan el trabajo de Emilia y su esposo, que fueron militantes activos antes y durante los años '70, y que los llevó al exilio. Estos elementos no pueden estar ausentes cuando pensamos los aportes de esta autora.

Ferreiro no sólo estudiaba la adquisición de la lectoescritura en los niños de determinadas edades, buscando entender la lógica de esa construcción, y comprendiendo que en cada niño ese proceso sigue un ritmo propio. Sino que, en el desarrollo de esas posibilidades, que eran valoradas en la época como una capacidad de cada individuo, Ferreiro observaba también la influencia de las desigualdades sociales. En aquellos niños que la psicología evaluaba como evidenciando problemas de aprendizaje porque no respondían al ritmo esperado, Ferreiro hace visibles serios condicionamientos sociales que permanecían invisibilizados desde cierta óptica.

Respecto del contexto social en que se situaba el trabajo de investigación de las autoras y su posterior publicación, el texto se presenta como documento de una época. La investigación realizada en la Argentina no pudo ver la luz en el país, y fue publicada en el extranjero; llegó luego a la Argentina de manera clandestina y rápidamente comenzó a ser leído y discutido en grupos de estudio restringidos. Estos avatares dibujan un escenario social convulsionado, atravesado por sucesivas crisis políticas y cambios de gobierno donde todo indica que la producción y circulación de los saberes no fue ajena a ese devenir socio-político. La producción investigativa y las elaboraciones teóricas del equipo de trabajo que conducía Ferreiro producían una ruptura con saberes y prácticas circulantes en la época. Durante la dictadura, tanto dentro como fuera de las universidades, Piaget fue uno de los autores puestos en cuestión, en algunos casos sus obras fueron retiradas de las bibliotecas, y en otros se permitía su circulación siempre que su visión estuviera restringida a una psicología evolutiva solidaria con las prácticas profesionales orientadas por la evaluación psicométrica y psicodiagnóstica.

En el terreno de la pedagogía y las ciencias de la educación, habían aparecido otras voces, que relacionaban a Piaget con las corrientes de las nuevas pedagogías y la alfabetización popular. Es justamente en este punto de articulación de los conceptos piagetianos y las prácticas pedagógicas antihegemónicas, donde podemos ubicar el trabajo de Emilia Ferreiro en esos años. 


\section{Materiales y métodos}

Desde el punto de vista metodológico, esta indagación considera el texto Los sistemas de escritura en el desarrollo del niño (Ferreiro \& Teberosky, 1979) como un producto histórico, y analiza tanto el contenido del mismo como su contexto de producción. Las técnicas de análisis de discurso sobre textos psicológicos considerados como documentos históricos permiten poner en valor su riqueza histórica, dado que condensan las impresiones de una época en base a las articulaciones que el autor realiza; como si cada texto representara un turno de intervención en un diálogo cultural más amplio (Bajtin, 1996). Rosa, Huertas y Blanco (1996) han señalado que a pesar de la importante base material que el análisis de textos y discursos representa para el análisis histórico y de los variados procedimientos que se disponen para ello, la historia de la psicología ha considerado el texto científico como un medio transparente, como un mero soporte para los conceptos que intenta transmitir, a los que trata como entidades significantes independientes de la forma expresiva del texto, la función social que cumplió, su significado en la cultura o la intención comunicativa del autor.

Nuestro análisis se articula con los estudios de recepción, al atender a las lecturas más eficaces, a las maneras en que se recibieron, transformaron y apropiaron teorías psicológicas en la Argentina de los años 1970 (Vezzetti, 2007). Asimismo, y en atención a la importancia concedida a las fuentes primarias en el estudio histórico de la psicología (Klappenbach, 2014), se ha considerado valioso tomar en cuenta el punto de vista de los participantes (Jacó-Vilela, EspíritoSanto, Degani-Carneiro, Goes, \& Vasconcellos, 2016), a través de comunicaciones personales con actores claves, recuperando también relatos de colaboradores y entrevistas realizadas a Ferreiro publicadas bajo diferentes formatos. Finalmente el enfoque metodológico se complementó con los aportes de la microhistoria (Ginzburg, 1994; Levi, 1993).

El análisis del texto de Ferreiro \& Teberosky (1979) ha implicado la descripción, explicación e interpretación del mismo, entendidos como momentos sucesivos que no buscan descubrir un significado verdadero (Rosa, Huertas, \& Blanco, 1996). La descripción se inició con la identificación del texto en sus categorías básicas: autor, título, medio en el que se publicó, lugar y fecha de publicación, estructura de la obra. Luego se trató de detectar lo que constituye el diálogo del texto -voces e interlocutores y las condiciones en que ocurre-, circunscribiendo los problemas en discusión. La estructura argumental del texto permitió reconocer la intención teórica de la autora, o primera línea del diálogo, la cual se apoyó en recursos demostrativos que reforzaban su intención, como son los datos de la pobreza y el fracaso escolar en América Latina. El estudio de la 
estrategia global de la argumentación se orienta a identificar la tradición de la que el texto depende y la audiencia a la que va dirigido, de allí que el momento de la explicación requirió poner el texto en relación con su tradición científica, con los modos de organización social que lo posibilitaron y con la vida de la autora. Esta dialogicidad textual implicó reconocer la dialogicidad histórica o externa del texto, esto es, las tradiciones o problemáticas dentro de las cuales el texto cobra sentido y la dialogicidad interna o textual que muestra los modos de expresión interna del diálogo con su tradición y marca la relevancia del problema abordado.

En cuanto al examen de los contextos del texto, se destaca el papel de la audiencia y el contexto social contemporáneo del texto, de allí que se consideraron los interlocutores hacia quienes estaba dirigido docentes y profesionales-, y el uso que ha recibido, sin descuidar el contexto social contemporáneo en el que se produjo dicho texto, esto es los acontecimientos sociales, políticos, económicos y culturales del momento, que no sólo enmarcan el texto sino que constituyen sus condiciones de posibilidad y contribuyen a explicar la génesis, la producción y aún el impacto del mismo.

En síntesis, se ha señalado que la función última del análisis es señalar la heurística positiva del texto, que intentamos poner de manifiesto, y que constituye el momento de la interpretación, la cual nunca es definitiva.

De acuerdo a este marco, nuestra presentación se organiza en tres partes: en primer lugar se presenta el texto de Ferreiro, sus antecedentes y la investigación que le dio lugar. Luego destacaremos las innovaciones que de acuerdo a nuestra lectura, este texto produjo en la época y que se encuentran vigentes aún hoy. Finalmente expondremos los avatares en la recepción del texto en Argentina, en el marco de la dictadura militar.

\section{La labor investigativa de Ferreiro en la década de 1970}

En este apartado presentaremos brevemente la formación profesional de Ferreiro, su trayectoria militante, el encuentro con Piaget. Describiremos también la investigación que posibilitó la publicación del libro y el contexto social en el cual este se produjo.

\section{Los comienzos}

Emilia Ferreiro nació en Buenos Aires en 1937, estudió psicología en la Universidad de Buenos Aires, y formó parte de la primera generación de psicólogas argentinas. En la década de 1960 se desempeñó como ayudante en Psicología General II, asignatura donde se estudiaba a Piaget (Ferreiro en Denti, 2008; Caruso \& 
Fairstein, 2003). En aquel período, la enseñanza de Piaget se afianzaba en las carreras de psicología y pedagogía de las Universidades Nacionales, y en general se lo introducía en el marco de una psicología evolutiva, signada por el orden de las etapas de adquisición de nuevas habilidades (Alvarez, Giordano, \& Vissani, 2013).

El encuentro personal de Ferreiro con Piaget se produjo a fines de la década de 1960, cuando se vió obligada a abandonar el país a consecuencia de la situación política y social que se vivía en la Argentina (Ferreiro en Denti, 2008). En 1966, luego del golpe de estado y la instalación del gobierno de facto del General Onganía, se produjo una brutal intervención al sistema científico argentino por la cual se desmantelaron abruptamente valiosos proyectos de investigación científica. Numerosos intelectuales y científicos fueron separados de las universidades en un violento episodio conocido como la Noche de los Bastones Largos (Morero, Eidelman, \& Lichtman, 2002). Por la presión política que vivía con su marido, el destacado físico Rolando García, Ferreiro debió partir al exilio. Realizó su doctorado en el Centro de Epistemología Genética de la Universidad de Ginebra sobre Las relaciones temporales en el lenguaje del niño, dirigida por el mismo Piaget.

Emilia Ferreiro regresó de Suiza en 1971, y se reincorporó a la Universidad de Buenos Aires. Allí reunió un equipo de colaboradoras con quienes inició en 1972 un trabajo de investigación sobre lectoescritura con niños provenientes de sectores de bajos recursos de las escuelas del conurbano bonaerense. Las colaboradoras fueron Ana Teberosky, Alicia Lenzi, Susana Fernández, Ana María Kaufman y Liliana Tolchinsky. Hasta 1974 la investigación era parte de sus tareas docentes en la Universidad de Buenos Aires, pero se les retiró el apoyo oficial luego que las universidades nacionales fueran nuevamente intervenidas (Rodríguez, 2015). Finalmente en 1976 el trabajo de investigación debió ser definitivamente interrumpido por la persecución política que se inició con la dictadura militar de 1976; las integrantes del equipo partieron al exilio, y cada una tomó caminos diferentes: Ana María Kaufman se radicó en México, Ana Teberosky en Barcelona, Emilia Ferreiro partió primero a Suiza y luego se instaló en México.

\section{La investigación}

Al poco tiempo de retomar su trabajo en la Universidad de Buenos Aires, Ferreiro comenzó a diseñar la investigación. Venía de Ginebra con el propósito de hacer estudios comparativos en psicolingüística, interesada en descubrir cómo se aprendían ciertas construcciones sintácticas y también en comprender un poco más las razones del fracaso escolar inicial, puesto que a comienzos de la década de los 
70 ese era uno de los mayores problemas del sistema educativo en América Latina (Ferreiro en Denti, 2008). La perspectiva piagetiana que había adquirido en Europa señalaba a la investigación empírica rigurosa como el camino para el progreso conceptual y teórico.

En la búsqueda de esas evidencias empíricas, y como paso previo a la preparación de situaciones experimentales, comenzó a observar clases en zonas marginadas de la ciudad de Buenos Aires, donde encontró una enorme cantidad de intercambios lingüísticos relacionados con la escritura. Recuperando la valiosa indicación de Piaget de considerar al niño como un sujeto cognoscente, -como alguien que trata de entender el mundo que le rodea, que elabora hipótesis, que recibe la información a su manera pues depende de los esquemas asimiladores con los que cuenta para interpretar esa información-, Ferreiro se plantea si sería posible encontrar ese niño piagetiano en el terreno de la lengua escrita (Ferreiro en Denti, 2008).

El grupo de investigación trabajó en escuelas primarias y jardines de infantes de sectores marginales de la ciudad de Buenos Aires y comenzó a encontrar de manera sistemática que aquellos niños que fracasaban en la escuela y repetían el curso una y otra vez, de alguna manera sabían lo que la escuela enseñaba. Esto es, que esos alumnos repitentes "sabían perfectamente bien todas las letras, sabían perfectamente bien la relación con los sonidos y no podían leer ni escribir" Kaufman (como citado en Aspis \& Horta, 2015).

La investigación requirió de la preparación de situaciones específicas, ya que los tests usuales que valoraban la maduración del niño para la lectoescritura, tal como el $A B C$ de Lourenço Filho, no eran útiles a los objetivos de ese trabajo, en el cual se trataba de que el niño ponga en juego la escritura y la lectura "tal como él las ve y explicite los problemas que se plantea" (Ferreiro \& Teberosky, 1979, p. 38). A partir de estas situaciones, se trataba de entablar un diálogo, siguiendo el método clínico de Piaget. Durante estas entrevistas individuales con los niños, se registraron las respuestas y cada sujeto era evaluado en toda la serie de tareas, en la escuela o jardín al que asistía. Se estudiaron durante un año 30 niños de primer grado de una escuela de un barrio periférico del gran Buenos Aires, realizando un seguimiento semi longitudinal (Ferreiro \& Teberosky, 1979, p.43). Los niños pertenecían a un medio social de clase baja, habitantes de lo que se denomina en Argentina una villa miseria, sus familias eran obreros no calificados o trabajadores temporarios. Los métodos de enseñanza que empleaban las maestras eran los mismos. Los resultados dejaron claro que los niños llegan a primer grado con toda un serie de hipótesis sobre la escritura, y que estas comienzan alrededor de los 4 años. 
El golpe de Estado de 1976 impidió que el grupo continuara trabajando, primero debieron abandonar la muestra de niños de escuelas urbano-marginales y luego partir al exilio.

\section{EI libro}

El texto comienza explicitando muchos de los aspectos que anticipan el impacto que el trabajo de Emilia Ferreiro produjo: la escritura como objeto cultural, la relación que entabla el niño con ese objeto que intenta asimilar, y su condición como sujeto cognoscente antes que como objeto de distintos métodos didácticos. Dice así:

“Un número muy importante (demasiado importante) de niños fracasan al ser introducidos a la alfabetización inicial. Pretendemos demostrar que el aprendizaje de la lectura, entendido como el cuestionamiento acerca de la naturaleza, función y valor de este objeto cultural que es la escritura, comienza mucho antes de lo que la escuela imagina, y procede por vías insospechadas. Que además de los métodos, de los manuales, de los recursos didácticos, existe un sujeto que trata de adquirir conocimiento, que se plantea problemas y trata de resolverlos siguiendo su propia metodología. (...) Un sujeto que la psicología de la lectoescritura ha olvidado, a fuerza de buscar aptitudes específicas, habilidades particulares, o una siempre mal definida madurez lograda." (Ferreiro \& Teberosky, 1979, p. 9)

En el campo específico del aprendizaje de la lectoescritura, produce una verdadera revolución, tanto en nuestro país como en otros países de América Latina, principalmente en México y Brasil (Aspis \& Horta, 2015). Publicado en México en 1979, con prólogo de la reconocida investigadora Hermine Sinclair, se recogen en el texto los resultados de la investigación realizada entre 1972 y 1975. Su lectura por parte de psicólogos y educadores, produjo un intenso cuestionamiento de las prácticas docentes derivadas de modelos tradicionales enseñanzaaprendizaje, y una reevaluación de las conceptualizaciones sostenidas desde la teoría de Piaget.

\section{Las innovaciones que introduce el texto}

De acuerdo a nuestra lectura del texto, hemos podido identificar cuatro dimensiones o ejes en la que dicha obra produce aportes al campo del aprendizaje de la lectoescritura y la investigación psicológica: 1) Introduce una lectura epistemológica de la teoría de Piaget, diferente a la mirada evolutiva que circulaba en las carreras 
de psicología en la Argentina en aquel momento; 2) Articula un desarrollo de los conceptos de la psicología genética en el campo de la lectoescritura, el cual no había sido abordado en los estudios piagetianos 3) Presenta estos conceptos como resultado de una investigación empírica efectuada en base al método piagetiano, lo cual era novedoso en la Argentina, 4) Incluye una comprensión del fracaso escolar en una perspectiva más amplia, que pudiera considerar aspectos hasta ese momento invisibilizados, tales como la desigualdad social y las condiciones de pobreza de América Latina.

\section{Una lectura epistemológica: El niño como sujeto cognoscente}

Emilia Ferreiro fue una de las pioneras, junto con Sara Paín, en incorporar al entramado de las lecturas locales el aporte de las últimas investigaciones del Centro de Epistemología Genética. Estas desplazaban el énfasis en las etapas del desarrollo del niño a las preguntas epistemológicas acerca de los mecanismos de adquisición y construcción del conocimiento en general y también del conocimiento científico (Caruso \& Fairstein, 2003). Se comenzaba a hacer hincapié en la actividad del sujeto que conoce, en la actividad del niño en la construcción del conocimiento, quien ya no será entendido como un receptor pasivo que recibe un conocimiento desde afuera (Castorina, Ferreiro, Oliveira, \& Lerner, 1996; Paín, 1997; Laino, 2000).

Las concepción dominante sobre el aprendizaje en la época estaba ligada a la maduración del niño, y consistía en una serie de habilidades específicas susceptibles de ser medidas a través de conductas observables, de allí la importancia que ese modelo concedió a la evaluación psicológica. Las descripciones del niño considerado normal, habían tomado un sentido prescriptivo, y transformadas en requisitos necesarios para acceder a la educación. En la literatura psicológica se establecía la lista de las aptitudes o habilidades necesarias para aprender a leer y escribir: lateralización espacial, discriminación visual, discriminación auditiva, coordinación visomotriz, buena articulación, entre otras (Ferreiro \& Teberosky, 1979 , p. 28). Esta caracterización del niño servía de base para una clasificación escolar implícita o explícita y constituía el fundamento de la respuesta que la psicología de la época daba al fenómeno del fracaso escolar. En general, la causa de ese retraso era atribuida al aspecto madurativo, respuesta de tipo biologicista en su explicación última. Ferreiro se separa tajantemente de las concepciones conductistas del aprendizaje, puesto que la concepción piagetiana se ocupa de un sujeto del aprendizaje que es productor de conocimiento (Ferreiro \& Teberosky, 1979, p. 36). 


\section{La perspectiva piagetiana en un nuevo campo: la lectoescritura}

Los estudios e investigaciones con niños realizados por Piaget en Ginebra - luego reproducidos por sus colaboradores en otros países del mundo- exploraban la construcción de distintas nociones en el pensamiento infantil en diferentes momentos. Estas nociones abordadas a partir del método clínico buscaban explicar la construcción de distintos objetos del universo lógico-matemático y físico: el objeto, el espacio, el tiempo, el número, la longitud, el peso, el volumen (Piaget, 1973, 1991). Sin embargo, a excepción del trabajo de Hermine Sinclair sobre el lenguaje, no se habían efectuado hasta ese momento investigaciones acerca de la lengua escrita desde la perspectiva de la psicología genética (Sinclair como citado en Ferreiro y Teberosky, 1979). Emilia Ferreiro indagó con ese método en un dominio que Piaget no había trabajado, el de la escritura entendida como un objeto cultural, y exploró la relación que el sujeto cognoscente puede establecer con ese objeto, tal como Piaget lo hacía respecto a la construcción de otros objetos. Para las autoras:

“lo que está en juego aquí es la concepción misma que se tiene acerca de la teoría de Piaget: o bien se la concibe como una teoría limitada a los procesos de adquisición de conocimientos lógico-matemáticos y físicos, o bien como una teoría general de los procesos de adquisición de conocimiento." (Ferreiro \& Teberosky, 1979, p. 31)

La perspectiva piagetiana, al explorar la adquisición de la lengua escrita por parte del niño, es muy diferente de la mirada tradicional sobre ese proceso. Las posiciones conductistas entendían el conocimiento como una copia pasiva del modelo externo (Burman, 1998). Estas explicaciones sobre la adquisición de la lengua escrita ponían el acento en las habilidades que debía poseer el sujeto para iniciar el proceso de adquisición de la lectoescritura y en la búsqueda de mejores métodos de enseñanza, trabajados desde la didáctica de manera externa al sujeto. El punto de partida de Ferreiro suponía una diferencia sustancial, la perspectiva piagetiana la conducía a indagar en la relación entre el sujeto y el objeto de conocimiento, dejando de lado los métodos de enseñanza. Desde esta perspectiva aparece una construcción epistémica diferente del objeto, así como nuevas hipótesis y conclusiones:

“Nuestra visión actual del proceso es radicalmente diferente: en lugar de un niño que espera pasivamente el reforzamiento externo de una respuesta producida poco menos que al azar, aparece un niño que trata activamente de comprender la 
naturaleza del lenguaje que se habla a su alrededor, y que, tratando de comprenderlo, formula hipótesis, busca regularidades, pone a prueba sus anticipaciones y se forja su propia gramática -que no es simple copia deformada del modelo adulto, sino creación original-. (Ferreiro \& Teberosky, 1979, p. 22).

Este contundente giro trata a la lectoescritura como un objeto a aprender y ya no como un objeto a enseñar, por lo cual influirá sobre las preguntas acerca de los métodos como sobre la posición del docente. Este ya no será aquel que aplica el método más eficaz, sino aquel que permite que los errores puedan ser reconocidos y trabajados como construcciones parciales, que van permitiendo aproximaciones a logros posteriores. No obstante, una suerte de dependencia de los métodos por parte del sistema educativo, produjo más tarde contradicciones en la interpretación y aplicación de las formulaciones de Emilia Ferreiro, que paradójicamente fueron entendidos y llevados a las aulas como métodos de enseñanza.

\section{El modelo piagetiano de investigación}

A su regreso a la Argentina, Emilia Ferreiro continuó trabajando a partir de los interrogantes generados luego de sus estudios en Suiza. La continuación de esos estudios en base al método piagetiano, se efectuó mediante la realización de una investigación en terreno, ya que como lo entendía Piaget, para que se produzca un progreso conceptual y teórico este debe necesariamente ser sostenido por evidencias empíricas rigurosas obtenidas en situaciones experimentales.

Tal como lo hemos descrito, el estudio fue minucioso y constituyó la base empírica que sostiene la idea de psicogénesis de la lectoescritura expuesta por las autoras en el libro. En él se describen distintas hipótesis que los niños elaboran al abordar este objeto a conocer y que permiten establecer ciertas regularidades. Estas se obtuvieron siguiendo el método clínico-crítico definido por Piaget, por el cual el investigador entabla un diálogo con el niño a partir de sus acciones, en el que busca reconocer los mecanismos estructurales presentes en estas.

Como modelo de investigación, este se vió interrumpido en su continuidad y desarrollo a partir del golpe de estado, en un momento en que el campo profesional de la psicología se encontraba en pleno crecimiento y efervescencia. Se suspendió así una tradición en ciernes que ubicaba a la investigación empírica desde una perspectiva diferente a las investigaciones que se estaban consolidando en la 
Argentina de acuerdo a tradiciones norteamericanas (Piñeda \& Jacó Vilela, 2014).

\section{EI fracaso escolar en perspectiva social}

Las autoras comienzan citando datos de la Unesco de 1974 sobre el fracaso escolar en América Latina, que ponían de manifiesto la gravedad de la situación en ese momento, y al mismo tiempo, presentaban el problema bajo otras coordenadas. Según esos datos, en 1970 el $20 \%$ de niños en edad escolar se encontraba fuera de la población escolarizada, y solo el $53 \%$ llegaba a cuarto grado, el resto abandonaba, por lo cual el mayor índice de repitencia se daba en los primeros grados de escolaridad. Esta preocupación por la situación educativa de grandes sectores de la población latinoamericana fue considerada bajo una mirada que permitía visibilizar la incidencia de las desigualdades sociales y económicas en el fracaso escolar. Desde un principio Ferreiro se aparta de las concepciones de su época en esta materia, que ponían el acento en las fallas del alumno o en los métodos de enseñanza, y que consideraban "males endémicos" del sistema educativo a la repitencia, el ausentismo y la deserción.

El análisis de las estadísticas les permitía concluir que estos problemas no estaban proporcionalmente repartidos en la población, sino que se acumulaban en determinados sectores que, por razones étnicas, sociales, económicas o geográficas, resultaban desfavorecidos. "Es entre la población indígena, rural o marginada de los centros urbanos, donde se concentran los mayores porcentajes de fracasos escolares" (Ferreiro \& Teberosky, 1979, p. 16) Las autoras se preguntaban: "¿cuál es la causa que hace que un individuo se convierta en repetidor, luego en desertor y acabe siendo subinstruido toda su vida?". E indicaban:

“...creemos que en lugar de "males endémicos", habría que hablar de selección social del sistema educativo; en lugar de llamar "deserción" al abandono de la escuela, tendríamos que llamarlo expulsión encubierta. $Y$ no se trata de un cambio terminológico, sino de otro marco interpretativo, porque la desigualdad social y económica se manifiesta, también, en la distribución desigual de oportunidades educativas" (Ferreiro \& Teberosky, 1979, p. 17)

Es interesante seguir el razonamiento que realizan las autoras a este respecto, lo hacen de una manera muy explícita y sencilla, analizando cada uno de los factores que en ese momento se entendían como causa del fracaso. Sobre el ausentismo escolar, si bien constatable, ellas se preguntan sobre las razones del mismo y lo relacionan 
directamente con las condiciones climáticas o de distancia que dificultan la llegada a la escuela, así como la necesidad frecuente de las familias de bajos recursos de contar con la ayuda de los niños para el sostén de la economía familiar.

El otro factor al que se alude es la repitencia, y allí su postura es terminante respecto de la falta de respuestas del sistema educativo en este tema. "Cuando un niño fracasa en el aprendizaje, la escuela le ofrece una segunda oportunidad: comenzar nuevamente el proceso de aprendizaje. ¿Es esta una solución?" (Ferreiro \& Teberosky, 1979, p.16). Las autoras indican que este tipo de propuesta educativa, al mantener las mismas condiciones, sólo ofrece al niño la posibilidad de repetir una experiencia de fracaso, por lo que el sistema de alguna manera expone al niño a un nuevo fallo.

Y finalmente, la contundencia de la reflexión sobre la deserción escolar, problema central del fracaso educativo: “El término "deserción" lleva implícita una carga significativa que supone la responsabilidad voluntaria del sujeto -en este caso, niños- al abandonar individualmente un grupo o sistema al que pertenece." Las autoras desplazan la responsabilidad que se le atribuía al niño y sus familias, y la dirigen hacia el sistema educativo, indicando que "es éste quien abandona al desertor, al no tener estrategias para conservarlo, ni interés en reintegrarlo." (Ferreiro \& Teberosky, 1979, p. 17). De allí es que podemos reconocer cómo el fracaso escolar es interpretado en clave social y no desde las supuestas dificultades o falta de maduración del niño.

Estas lecturas de la teoría piagetiana se recuperaron más tarde, con el retorno del sistema democrático a la Argentina, permitiendo reabrir un campo profesional para aquellos psicólogos que continuaban pensando la profesión en términos de cambio social posible. En Córdoba, este movimiento produjo cambios en la política educativa que el primer gobierno democrático impulsó desde 1983, en la Reforma Educativa de Córdoba entendida como un programa que intentó condensar un discurso político con uno pedagógico, y ocuparse desde lo educativo de grandes sectores postergados de la población (Abratte, 1999).

\section{La recepción del texto}

Nuestro interés por el texto de Emilia Ferreiro y Ana Teberosky (Ferreiro \& Teberosky, 1979) se incrementó a partir del impacto que una profesional nos transmitió en una entrevista. Actualmente profesora universitaria, esta profesional fue docente en el nivel primario a fines de la década de 1960, y nos relató su encuentro con el libro así como el efecto que este le produjo, tanto en sus concepciones sobre el niño que aprende como en sus prácticas 
docentes. La anécdota que nos refirió en esa oportunidad resulta muy interesante dado que revela algunas de las vías de circulación del conocimiento en condiciones de clandestinidad. Ella refiere que ese texto le fue regalado en 1979, el mismo año de su publicación, por un amigo que vivía en México, quien le envía un libro cuyas tapas estaban cubiertas con otro papel para ocultar el autor y le dice: "acaba de salir un libro en México que se está vendiendo como el pan, es una investigación reciente pero que comenzó en Argentina, es el primer libro de Ferreiro, seguro a vos te va a interesar" (M. A. M., comunicación personal, septiembre 2014). Indica cómo le impactó su lectura, que inmediatamente comenzó a entender el proceso de escritura de cada uno de los niños, en qué momento de la construcción estaba cada uno, y que las dificultades no remitían a algún tipo de retraso, es decir, que desde ese momento podía explicarse de otro modo qué pasaba con algunos de sus alumnos que fracasaban (M. A. M., comunicación personal, septiembre 2014).

Un impacto semejante encontramos en una entrevista publicada por Delia Lerner, colaboradora de Emilia Ferreiro durante los años ' 70. Dice Lerner:

“...durante un año hicimos un grupo de estudio interno sobre el libro y lo primero que yo me acuerdo de esa situación, es de una de las personas en el equipo que había sido mucho tiempo maestra de primer grado, que a cada momento se agarraba la cabeza diciendo: qué hice, qué hice, o sea: el impacto, la fuerza de una visión completamente diferente de la visión que uno tenía de ese chico que estaba aprendiendo a leer y escribir, y que de pronto podía ser que tuviera ideas propias totalmente distintas de las que los métodos de enseñanza estaban tratando de implantar." (Lerner como citado en Aspis \& Horta, 2015).

En esas dos anécdotas se pone de manifiesto el efecto subjetivo que la lectura de este libro tuvo en educadores y psicólogos que trabajaban con niños con dificultades de aprendizaje. En un momento en el que la dictadura militar imponía severas restricciones a algunos temas y autores, estos profesionales podían advertir pero no manifestar su desacuerdo con las explicaciones vigentes sobre el fracaso escolar, y a la vez no disponían de conceptualizaciones rigurosas para considerar de otra manera el fenómeno con el que se encontraban en sus prácticas cotidianamente. Consideramos que es en este aspecto donde radica la fuerza del trabajo de Emilia Ferreiro, el cual dio lugar a nuevas lecturas, grupos de discusión, y diversas experiencias en relación al aprendizaje de la lectoescritura. El estudio de la recepción del texto nos llevó a analizar una historia de las lecturas más eficaces, y a prestar atención a los contextos de 
apropiación de los saberes circulantes (Vezzetti, 2007; Fantini, Vissani, \& Scherman, 2018).

La revalorización de estas anécdotas permite poner en primer plano lo acontecido en aquel momento como objeto de un análisis en el que identificamos distintas dimensiones y vectores, en vistas a la construcción de una historia de la psicología más rica y plural. Los postulados de la microhistoria como enfoque historiográfico, autorizó un modo de aproximación basado en la reducción de la escala de observación, y permitió prestar atención a lo pequeño, a lo singular, de manera de considerar dimensiones no advertidas anteriormente. Para Ginzburg (1994), las investigaciones de microhistoria y de historia local apuestan a mejorar la comprensión de la relación de los eventos con el contexto; el autor plantea una posición política e historiográfica donde la mirada cercana permite atrapar lo que escapa a una visión global. En la práctica de reducción de la escala se destaca el valor de la mirada y el estudio intensivo del material documental como aspecto esencial de lo micro. La investigación microhistórica permite insertar acciones más nimias en sistemas más generales (Levi, 1993). Ocampo López (2007) reivindica lo local como fuerza constituyente de la identidad y enfatiza la importancia de las microhistorias como camino al reconocimiento de una historia que reivindique la fortaleza política de lo regional.

\section{Discusión}

La investigación realizada, que debió interrumpirse, primero por falta de apoyo oficial y luego porque las investigadoras debieron abandonar el país debido a la persecución política, produjo con la publicación del texto tres años después, un importante cuestionamiento de las prácticas docentes y psicológicas ligadas al campo educativo, como una ruptura con perspectivas teóricas tradicionales sobre el aprendizaje y el fracaso escolar.

El texto de Ferreiro (1979) trajo aparejado varias novedades: una nueva lectura epistemológica de la teoría de Piaget, en la cual se pusieron en juego avances teóricos del autor en el campo de la lectoescritura. A la vez, se realizó una investigación empírica en base al método piagetiano, la cual fue efectuada en sectores vulnerables de la población, y se puso en primer plano las desigualdades sociales y políticas del contexto educativo en América Latina como explicación del fenómeno del fracaso escolar.

Es una afirmación que nos permite inferir que probablemente estas conceptualizaciones que Ferreiro transmite son factores que contribuyeron a que Piaget fuera asociado a un pensamiento cuestionador de las desigualdades sociales y a prácticas 
transformadoras de la realidad social, y en consecuencia se viera restringida su circulación durante la dictadura.

La aproximación desde la microhistoria nos permitió revalorizar el relato de una anécdota que nos condujo a la revisión detallada de un texto que se constituye en expresión de una época, en el cual a la vez que se formula teoría científica, se condensan elementos de múltiples dimensiones: epistémicas, sociales, políticas y económicas. En el texto está presente la convicción de producir teoría científica para el cambio social y político en Latinoamérica; en la recepción del mismo aparecen los determinantes del contexto social impuesto por la dictadura militar, como el exilio, la censura y la clandestinidad. Se manifiestan también los intereses intelectuales de una generación profesional, la manera en que nuevos conocimientos impulsaron una reevaluación de las prácticas profesionales. Finalmente esta producción reafirma una lectura de Piaget que ubica al niño como un sujeto cognoscente en el dominio de la lectoescritura, que fue dando lugar a nuevas prácticas y maneras de mirar a los niños.

Finalmente, la manera en que se produjo el encuentro con el texto de Ferreiro se enlaza con el problema de la persecución ejercida sobre los intelectuales por la dictadura militar y las formas clandestinas de circulación de saberes al que una generación profesional se enfrentó. Hemos señalado que estos razonamientos expresados en el texto, este decir que llamaba a una transformación de esa realidad, no podían ser expresados abiertamente en la segunda mitad de la década del 70, luego del golpe de estado. Incluso podría señalarse que el mismo hacer de estas investigadoras y docentes universitarias, el hecho de dirigirse a las escuelas de los sectores marginados, ya era una transformación en sí. Implicaba, como acto, un cambio en la posición y en la mirada desde el lugar del intelectual, del universitario, del psicólogo.

\section{Referencias}

Abratte, J. P. (1999). Una aproximación al discurso político-educativo de la Reforma Educacional de Córdoba. Cuadernos de Educación, 2(2) 39-49.

Alvarez, S., Giordano, S., \& Vaissni, L. (2013). La psicología del niño en la carrera de psicología de la UNC. Autores y contenidos predominantes. Actas del Encuentro Argentino de Historia de la Psiquiatría, la Psicología y el Psicoanálisis (vol. 14, pp. 352358). San Miguel de Tucumán: Universidad Nacional de Tucumán, 14.

Aspis, P., \& Horta, R. (2015). Serie Educadores: Emilia Ferreiro. Concurso Público 2015, Prefeitura Duque de Caixas. Municipio Duque de Caixas. RJ. Brasil. 
Bajtín, M. (1996). La imaginación dialógica. Buenos Aires: Taurus.

Burman, E. (1998). La deconstrucción de la psicología evolutiva. Madrid: Visor.

Caruso, M., \& Fairstein, G. (2003). Las puertas del cielo. Hipótesis acerca de la recepción de la psicogénesis y el constructivismo de raíz piagetiana en el campo pedagógico. In A. Puiggrós (Dir.), Dictaduras y utopías en la historia reciente de la educación argentina (1955-1983). Buenos Aires: Galerna.

Casanova, J. (2015). La historia social y los historiadores. Barcelona: Planeta.

Castorina, J. A., Ferreiro, E., de Oliveira, M. K., \& Lerner, D. (1996). Piaget-Vigotsky: contribuciones para replantear el debate. Paidós Educador.

Denti, J. [TVAL Producciones. Canal 22 y Canal Encuentro]. (2008, Marzo 26). Los Argenmex. Emilia Ferreiro [Arquivo de vídeo]. México, D.F.: Canal Encuentro. Retirado de https: //www.educ.ar/recursos/102321/emilia-ferreiro

Fantini, N., Vissani, L., \& Scherman, P. (2018). Atravesamientos del contexto socio-histórico en la construcción del rol profesional del psicólogo. In M. Bricca (Ed.), Investigar en Ciencias Humanas hoy (pp. 52-66). Córdoba: EDUCC-Universidad Católica de Córdoba.

Ferreiro, E., \& Teberosky, A. (1979). Los sistemas de escritura en el desarrollo del niño. México: Siglo XXI Editores.

Ginzburg, C. (1994). Microhistoria: dos o tres cosas que sé de ella. Manuscrits: revista d'història moderna, (12), 13-42.

Grez Toso, S. (2005). Escribir la historia de los sectores populares. ¿ Con o sin la política incluida?. A propósito de dos miradas a la historia social. Política, 44, 17-31. doi:10.5354/07161077.2012.25600

Jacó-Vilela, A. M., Espírito-Santo, A., Degani-Carneiro, F., Goes, L., \& Vasconcellos, M. (2016). Investigando em História da Psicologia: contribuições metodológicas. Interacciones, 2(2), 123-134.

Klappenbach, H. (2003). La globalización y la enseñanza de la psicología en Argentina. Psicología em estudo, 8(2), 3-18.

Klappenbach, H. A. (2006). Periodización de la psicología en Argentina. Revista de Historia de la Psicología, 27(1), 109-164.

Klappenbach, H. (2014). Acerca de la Metodología de Investigación en la Historia de la Psicología. Psykhe (Santiago), 23(1), 01-12.

Laino, D. (2000). Aspectos psicosociales del aprendizaje. Rosario: Homo Sapiens Ediciones.

Levi, G. (1993). Sobre Microhistoria. In P. Burke et al., Formas de hacer historia. Madrid: Alianza Editorial.

Macchioli F., García, L., \& Talak, A. M. (2017). Itinerarios de la psicología. Buenos Aires: Miño y Dávila Eds. 
Morero, S., Eidelman, A., \& Lichtman, G. (2002). La noche de los bastones largos. Buenos Aires: Nuevohacer Grupo Editor.

Ocampo López, J. (2007). La microhistoria en la historiografía general. Revista Latinoamericana de Estudios Educativos (Colombia), 3(1), 9-26.

Paín, S. (1997). Psicopedagogía Operativa. Buenos Aires: Nueva Visión.

Piaget, J. (1973). La formación del símbolo en el niño. México: Fondo de Cultura Económica.

Piaget, J. (1991). Seis estudios de psicología. España: Editorial Labor.

Piñeda, M. A., \& Jacó-Vilela, A. M. (2014). Ciencia psicológica y profesionalización en Argentina y Brasil: 1930-1980. Universitas Psychologica, 13(4), 15-32. doi:10.11144/Javeriana.upsy135.cppa

Rodríguez, L. G. (2015). La universidad durante el tercer gobierno peronista (1973-1976). Conflicto social, 7(12), 114-145.

Rosa, A., Huertas, J. A., \& Blanco, F. (1996). Metodología para la historia de la psicología. Madrid: Alianza Editorial.

Vezzetti, H. (2007). Historias de la psicología: problemas, funciones y objetivos. Revista de Historia de la Psicología, 28(1), 147-166.

\section{Endereço para correspondência \\ Patricia Scherman}

Universidad Nacional de Córdoba - UNC

Instituto de Investigaciones Psicológicas (IIPsi), Facultad de Psicología

Av. Enrique Barros, s/n, Ciudad Universitaria, CP.5000, Córdoba, Argentina

Endereço eletrônico: patoscherman@gmail.com

\section{Laura Vissani}

Universidad Nacional de Córdoba - UNC

Escuela de Ciencias de la Educación, Facultad de Filosofía y Humanidades (FFyH)

Av. Haya de la Torre, s/n, Ciudad Universitaria, CP.5000, Córdoba, Argentina

Endereço eletrônico: lauravissani@live.com.ar

\section{Nilda Fantini}

Universidad Nacional de Córdoba - UNC

Instituto de Investigaciones Psicológicas (IIPsi), Facultad de Psicología

Av. Enrique Barros, s/n, Ciudad Universitaria, CP.5000, Córdoba, Argentina

Endereço eletrônico: nildafantini@hotmail.com

Recebido em: 10/12/2018

Reformulado em: 18/02/2019

Aceito em: 28/02/2019

\section{Notas}

* Doctora en Psicología, Profesora Titular Facultad de Psicología, UNC.

** Lic. en Psicología, Profesora Asistente - UNC.

*** Lic. en Psicología, Maestranda en Antropología - UNC.

Financiamiento: Secretaria de Ciencia y Técnica de la Universidad Nacional de Córdoba (Secyt-UNC). 
Patricia Scherman, Laura Vissani, Nilda Fantini

Este artigo de revista Estudos e Pesquisas em Psicologia é licenciado sob uma Licença Creative Commons Atribuição-Não Comercial 3.0 Não Adaptada. 